\title{
Effects of Electrical Muscle Stimulation for Preventing Deltoid Muscle Atrophy after Rotator Cuff Repair: Preliminary Results of a Prospective, Randomized, Single-blind Trial
}

\author{
Goo Joo Lee, Hangyeol Cho, Byung-Hyun Ahn ${ }^{1}$, Ho-Seung Jeong ${ }^{1 匹}$ \\ Departments of Rehabilitation Medicine and ${ }^{1}$ Orthopedic Surgery, Chungbuk National University Hospital, Cheongju, Korea
}

\begin{abstract}
Background: This study investigates the effects of neuromuscular electrical stimulation (NMES) in preventing deltoid atrophy during the first 12 weeks after arthroscopic rotator cuff repair.

Methods: Eighteen patients undergoing arthroscopic repair of a medium-sized rotator cuff tear by a single surgeon, were randomized into two groups: NMES and transcutaneous electrical nerve stimulation (TENS). Each group used the respective device for 6 weeks after surgery. Pain was measured at baseline, 6 , and 12 weeks postoperatively, using the visual analogue scale (VAS); range of motion (ROM), abduction strength and functional scores were measured at baseline and 12 weeks postoperatively. Deltoid thickness and cross-sectional areas were measured using magnetic resonance imaging at 12 weeks postoperatively.

Results: At 12 weeks post-surgery, no statistically significant difference was observed between the NMES and TENS groups in the pain VAS, the Disabilities of the Arm, Shoulder and Hand score, ROM, and abduction strength. Postoperative decrease in the thickness of the anterior, middle, and posterior deltoid, at the level just below the coracoid, was $-2.5 \%,-0.7 \%$, and $-6.8 \%$, respectively, in the NMES group, and $-14.0 \%,-2.6 \%$, and $-8.2 \%$, respectively, in the TENS group $(p=0.016, p=0.677$, and $p=0.791$, respectively). At the level of the inferior glenoid tubercle, postoperative decrease in area of the deltoid was $-5.4 \%$ in the NMES group and $-14.0 \%$ in the TENS group, which was significantly different $(p=0.045)$.

Conclusions: NMES has the potential for reducing deltoid atrophy after arthroscopic rotator cuff repair, suggesting that NMES might help minimize postoperative atrophy after various shoulder surgeries.
\end{abstract}

(Clin Shoulder Elbow 2019;22(4):195-202)

Key Words: Shoulder; Rotator cuff; Deltoid muscle; Muscular atrophy; Electric stimulation

\section{Introduction}

An aging population and advancements in arthroscopic techniques have led to a steady increase in the number of repairs performed annually. There are approximately 300,000 cases of rotator cuff tear per year worldwide, making rotator cuff repair one of the most common orthopedic surgeries." Although rotator cuff repair generally improves pain and function, the complication rate is high. ${ }^{2}$ The most common complications are retear and stiffness, and several studies have also focused on atrophy of the shoulder muscles. ${ }^{3-8)}$ Atrophy of the deltoid muscle, especially that of its anterior fibers, makes shoulder flexion difficult since no other muscle can effectively compensate for its functional loss. ${ }^{9}$ A previous study reported postoperative decrease in the thickness of the deltoid, with no recovery 1 year later. ${ }^{7,8)}$ However, there is no report on the prevention of deltoid atrophy. Neuromuscular electrical stimulation (NMES) elicits skeletal muscle contractions through percutaneous electrodes that de-

Received August 19, 2019. Revised September 24, 2019. Accepted October 27, 2019.

Correspondence to: Ho-Seung Jeong

Department of Orthopedic Surgery, Chungbuk National University Hospital, 776, 1sunhwan-ro, Seowon-gu, Cheongju 28644, Korea Tel: +82-43-269-6077, Fax: +82-43-269-6359, E-mail: hoseung1@gmail.com, ORCID: https://orcid.org/0000-0001-7297-5534 IRB approval: Chungbuk National University Hospital (No. CBNUH 2018-04-007).

Financial support: This work was supported by the research grant of the Chungbuk National University Hospital in 2018. Conflict of interests: None. 
polarize underlying motor nerves. NMES using percutaneous electrodes is noninvasive and easy-to-use, and has reportedly shown good results in preventing muscle weakness and decreasing the thickness of the quadriceps muscle after anterior cruciate ligament surgery. ${ }^{10-15)}$ We therefore hypothesized that NMES could prevent deltoid muscle atrophy after arthroscopic rotator cuff repair, and performed a single-blind randomized study to compare the decrease in deltoid thickness in the NMES group and the transcutaneous electrical nerve stimulation (TENS) group (control).

\section{Methods}

\section{Patient Selection}

This investigation conformed to the principles outlined in the Declaration of Helsinki. All patients provided informed consent prior to experimentation. The study protocol was approved by the Medical Ethics Committee of our hospital. This study was a prospective, randomized, controlled trial. The inclusion criteria were medium-sized rotator cuff tears (tear size, 1-3 cm), planned arthroscopic repair surgery, and patient age between 40 and 75 years. Tear size was initially measured in preoperative magnetic resonance imaging (MRI), and confirmed arthroscopically using a calibrated probe at the time of surgery.

The exclusion criteria were incomplete repair of the footprint, preoperative fatty degeneration of the deltoid muscle, poor understanding of the stimulation devices (NMES or TENS), and refusal to participate in the study.

Sample size was determined by $\mathrm{G}$ power analysis (alpha error: 0.05 , power: 0.80 , calculated actual power: 0.82 ); a total of 22 patients were initially recruited as candidates for the study, 4 of whom were excluded due to preoperative fatty degeneration of the deltoid muscle $(n=1)$ and poor understanding of the stimulation devices $(n=3)$. Finally, 18 patients were enrolled between May 2018 and December 2018. Patients were assigned to either the NMES or TENS group, with a 1:1 allocation ratio based on the allocation sequence yielded by a computer-based block randomization process (block size, 4). Clinical and radiological evaluations were included in the follow-up at 12 weeks after surgery. The 3-month follow-up MRI was refused by 2 patients (NMES 1, TENS 1); thus, 8 patients in the NMES group and 8 in the TENS group were available for the final review (Fig. $1)^{16}$. Preoperative clinical data did not differ between the two groups (Table 1).

\section{Surgical Procedures}

Surgical procedures were performed by a single surgeon.

Table 1. Preoperative Data on Patients

\begin{tabular}{lccc}
\hline \multicolumn{1}{c}{ Variable } & NMES group & TENS group & p-value \\
\hline No. of patients & 8 & 8 & \\
Male/female & $5 / 3$ & $2 / 6$ & 0.131 \\
Mean age (yr) & $62.0(49-75)$ & $62.6(52-73)$ & 0.878 \\
Dominant/nondominant & $6 / 2$ & $7 / 1$ & 0.522 \\
shoulder & & & \\
Mean tear size (mm) & $19.1(11-29)$ & $21.4(13-29)$ & 0.887 \\
ROM (degrees) & & & \\
\hline \multicolumn{1}{c}{ Forward Flexion } & $154.4 \pm 32.7$ & $173.8 \pm 9.2$ & 0.145 \\
\multicolumn{1}{c}{ External Rotation } & $73.1 \pm 25.5$ & $86.9 \pm 8.8$ & 0.185 \\
\hline \multicolumn{1}{c}{ Abduction } & $143.8 \pm 39.6$ & $169.4 \pm 19.7$ & 0.171 \\
Pain VAS & $6.2 \pm 0.4$ & $6.1 \pm 0.4$ & 0.915 \\
\hline DASH score & $40.7 \pm 21.9$ & $27.9 \pm 14.5$ & 0.274 \\
\hline Peak torque, abduction (Nm) & $9.2 \pm 10.7$ & $12.5 \pm 14.0$ & 0.615 \\
\hline
\end{tabular}

Values are presented as number only, mean (range), and mean \pm standard deviation.

NMES: neuromuscular electrical stimulation, TENS: transcutaneous electrical nerve stimulation, ROM: range of motion, VAS: visual analogue scale, DASH: Disabilities of the Arm, Shoulder and Hand.

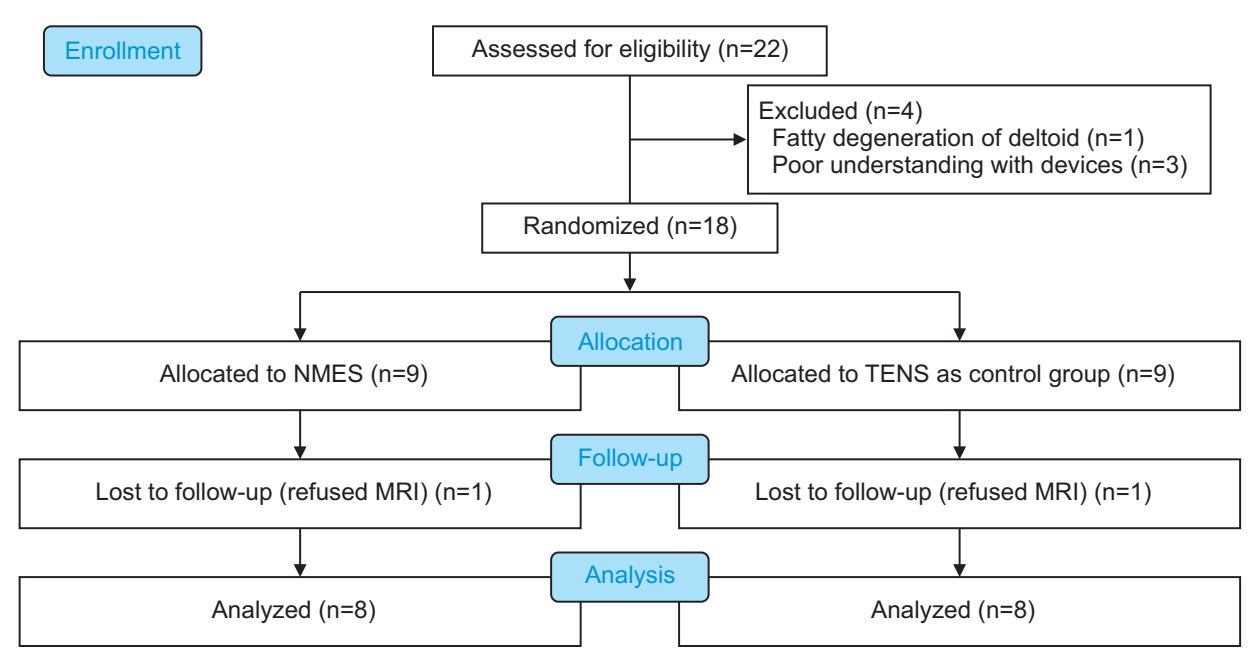

Fig. 1. Flow diagram: consolidated standards of reporting trials (CONSORT). ${ }^{16)}$

NMES: neuromuscular electrical stimulation, TENS: transcutaneous electrical nerve stimulation, MRI: magnetic resonance imaging. 
Under general anesthesia, patients were placed in the beachchair position. A posterior portal was first established for the evaluation of the glenohumeral joint. The associated biceps tendon lesion was treated by tenotomy ( 2 patients) in cases with subluxation or tear involving $>50 \%$ of the long head biceps. The arthroscope was subsequently removed from the glenohumeral joint and redirected into the subacromial space. The bursal tissue was removed for space clearance, and arthroscopic subacromial decompression was routinely performed to create a flat acromial undersurface in all patients. The footprint was prepared by removing the soft tissue and multiple channeling. The tear size was measured intraoperatively using a calibrated probe, after debridement of the degenerated tendon edges. All torn tendons were repaired using the suture bridge technique.

\section{Postoperative Rehabilitation and Intervention}

All patients underwent the same rehabilitation regimen. Briefly, shoulders were immobilized for 6 weeks with an abduction brace. Range of motion (ROM) exercises for the elbow, wrist, and fingers were started immediately after surgery. Pendulum exercise, passive forward elevation exercise, and shrugging of both shoulders were encouraged immediately after surgery. Active assisted ROM exercises were allowed after the patients were weaned off the brace. Muscle strengthening commenced at 12 weeks after surgery.

A portable EMS (EMS-1000; Cybermedic, Iksan, Korea) was used for the NMES intervention. This stimulator is practical for home use. During stimulation, the upper limb is secured by an abduction brace. Self-adherent, flexible rectangular electrodes are placed on the lower anterior and posterior portions of the deltoid muscle, and are marked to ensure consistent reapplication by the participant (Fig. 2).

NMES from the portable electrical stimulator was applied to the resting muscle, and the participant was instructed to relax during the induced muscle contraction. The intensity was set to the maximal intensity tolerated during each session, and partici- pants were repeatedly encouraged to increase the intensity as tolerated. The stimulator was set to deliver a biphasic current, using a symmetrical waveform, at $35 \mathrm{~Hz}$ for 5 seconds (including a 2.5 seconds ramp-up time and a 2 seconds ramp-down time) and a 5 seconds off time with pulse duration of $250 \mu$ seconds.

A portable TENS (KD-PRO5000; Kukdong Electronics, Ansan, Korea) was used for the TENS group. Active TENS units delivered a balanced, asymmetric, biphasic waveform with alternating pulses between channels. Similar to prior studies, the TENS units used a continuous frequency of 5 to $58 \mathrm{~Hz}$ for 60 seconds with pulse duration of $150 \mu$ seconds. ${ }^{17)}$ Patients were advised to adjust the intensity of the TENS unit to the highest tolerable setting that did not exacerbate their pain.

These interventions were initiated 24 hours after surgery, in patients assigned to the respective NMES and TENS groups. NMES or TENS were performed during each session, once daily, for 6 weeks after rotator cuff repair. An emphasis was placed on the importance of using the stimulator at a tolerable but slightly uncomfortable intensity, although there was no minimum intensity required for the study protocol. Moreover, the participants were repeatedly instructed to continue increasing to a tolerable intensity within and between sessions. Most participants demonstrated safe and proper use of the stimulator in the hospital. All patients visited the hospital 2 weeks after surgery, and we verified to confirm appropriate use as instructed.

\section{Clinical Evaluation}

The clinical assessment included ROM (forward flexion, external rotation, and abduction), pain visual analogue scale (VAS), deltoid muscle power (peak torque at abduction) and functional score (the Disabilities of the Arm, Shoulder and Hand [DASH] score, range: 0-100). These outcome measures were evaluated preoperatively, postoperatively at 6 weeks (pain VAS, DASH), and 12 weeks (pain VAS, ROM, DASH, and peak torque at abduction). The strength of shoulder abduction muscle was measured thrice by peak torque using Biodex (Biodex Corp., Shirley,
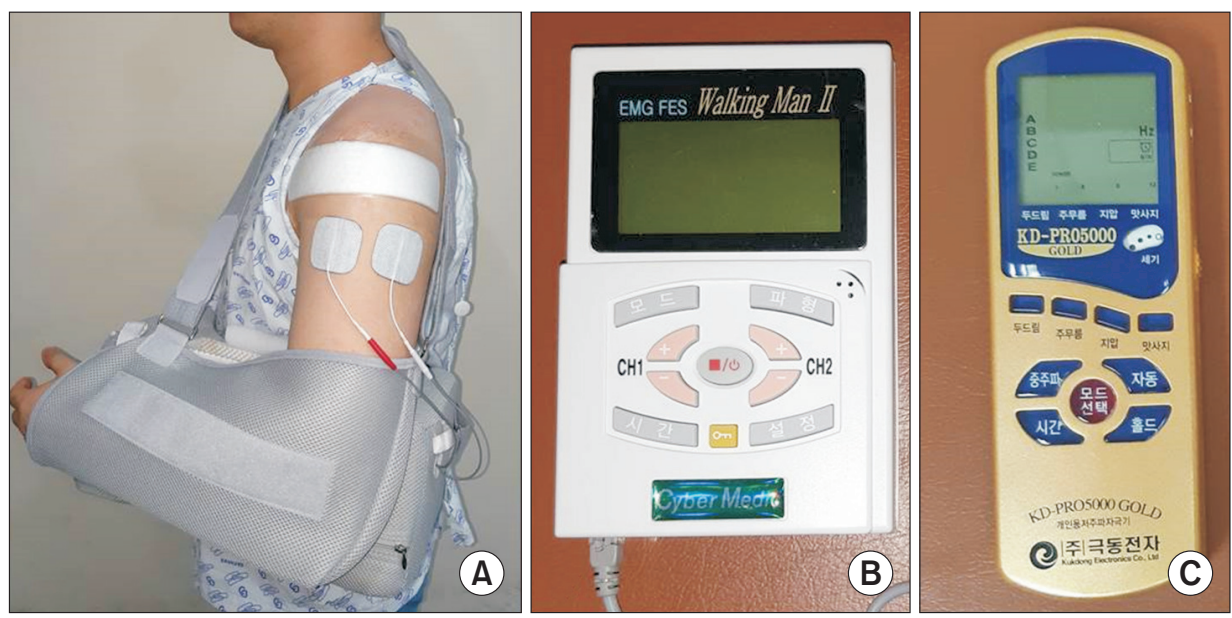

Fig. 2. Patient with stimulation device: (A) flexible rectangular electrodes were placed on the lower anterior and posterior portions of the deltoid muscle with abduction brace. (B) Neuromuscular electrical stimulation (NMES) devices were used in NMES group. (C) Transcutaneous electrical nerve stimulation (TENS) devices were used in TENS group. 
NY, USA), for 5 seconds at 60 degrees of shoulder abduction.

\section{Radiological Evaluation}

Anatomic evaluation of the rotator cuff and the deltoid muscle was achieved by MRI, because this method provides the benefit of multiplanar imaging of the shoulder. ${ }^{18)}$ All MRI studies were done with a 3.0-T unit (Achieva TX; Philips Medical System, Eindhoven, the Netherlands) and a dedicated shoulder array. Patients underwent imaging in the supine position, with the examined arm alongside the body in slight external shoulder rotation with the palm facing upward. Patients underwent MRI scans preoperatively and at 12 weeks postoperatively. Two orthopedic surgeons independently performed the MRI analysis in a randomized blind fashion. Measurements were performed on MRI images using the Picture Archiving and Communication System (PACS; Maroview ver. 5.4; MAROTECH Inc., Seoul, Korea).

The area of the deltoid muscle and the thickness of the ante- rior, lateral, and posterior deltoids were measured to compare the preoperative and postoperative deltoid volume, using the axial T2-weighted MRI images at the level just below the coracoid process (Fig. 3). Line 1 was drawn from the anterior edge to the posterior edge of the glenoid. Line 2 was drawn parallel to line 1 and passing the center of the humeral head, and the anterior and posterior deltoid thickness was measured along line 2 at the level just below the coracoid process. Line 3 was drawn perpendicular to line 1, and also passing through the center of the humeral head. Lateral thickness was measured along line 3. The cross-sectional area was measured on the same axial image (Fig. 3). The cross-sectional area of the deltoid muscle was measured at the level of the inferior glenoid tubercle using PACS (Fig. 4).

\section{Statistical Analysis}

A paired t-test was performed to assess differences in the preoperative and postoperative parameters of the deltoid muscle and the clinical outcomes of each group. An independent t-test
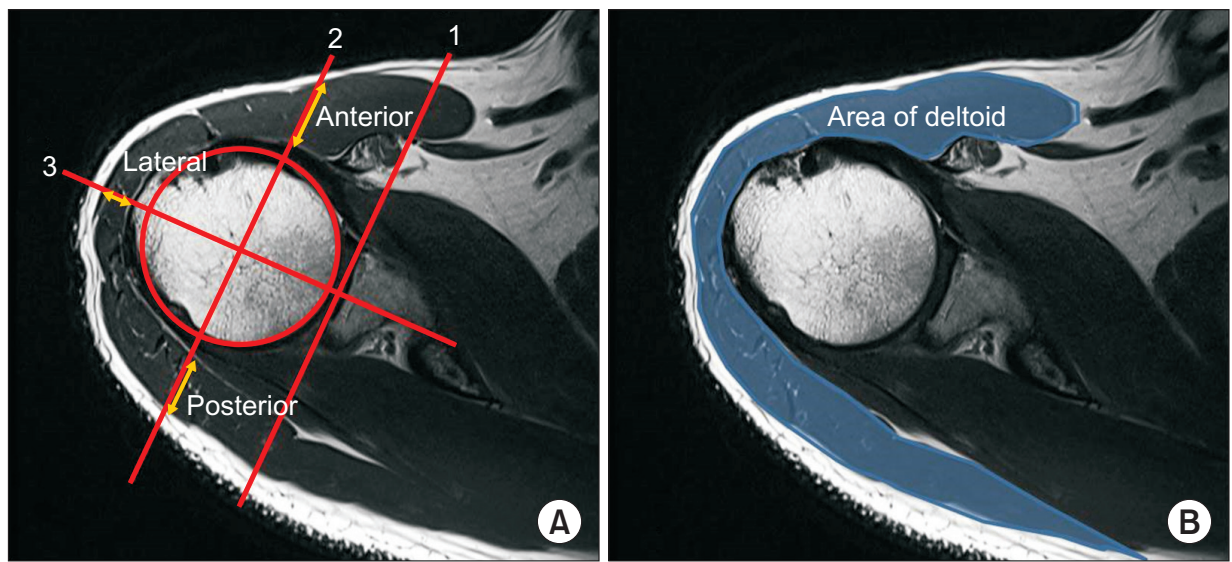

Fig. 3. Measurement of the deltoid thickness and the area at the level just below the coracoid process. (A) The thickness of the anterior, lateral, and posterior deltoids was measured using the axial T2-weighted magnetic resonance images (MRI) at the level just below the coracoid process. Line 1 was drawn from the anterior edge to the posterior edge of the glenoid. Line 2 was drawn parallel to line 1 and passing the center of the humeral head, and the anterior and posterior deltoid thickness was measured along line 2 at the level just below the coracoid process. Line 3 was drawn perpendicular to line 1 and also passes through the center of the humeral head. Lateral thickness was measured along line 3. (B) Cross-sectional area was measured on the same axial image.

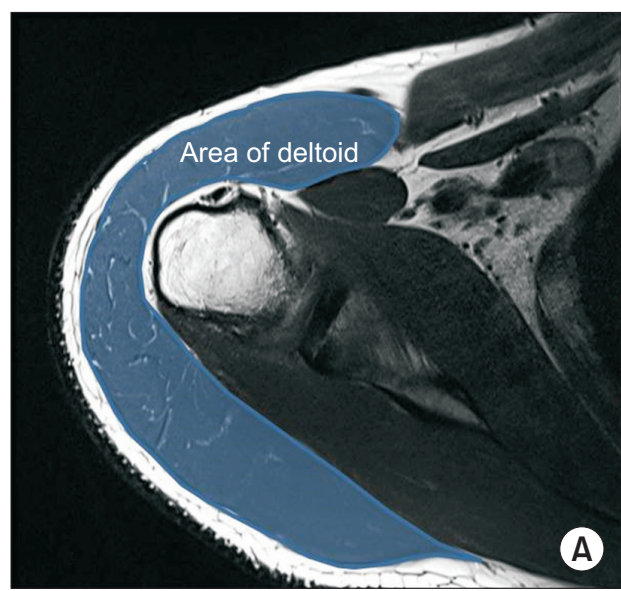

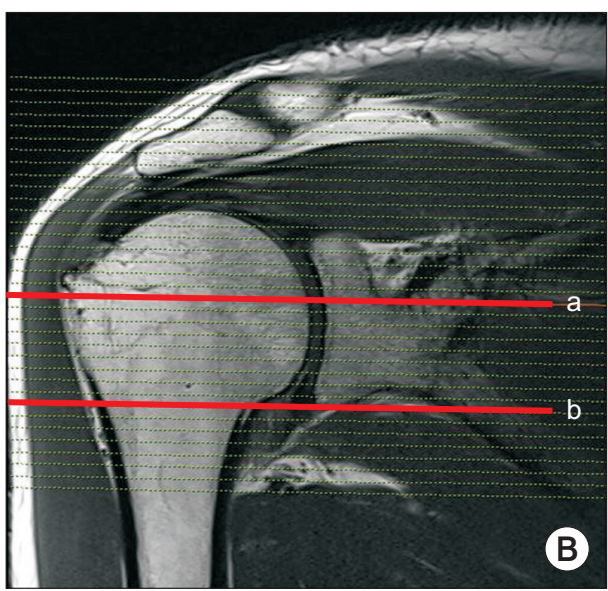

Fig. 4. Measurement of cross-sectional area of the deltoid thickness (at the level of inferior glenoid tubercle). (A) Cross-sectional area of deltoid was measured on axial T2weighted magnetic resonance imaging (MRI) at the level of inferior glenoid tubercle. (B) Cross-sectional line a showed the level just below the coracoid, and line b showed the level of inferior glenoid tubercle. 
was applied to compare postoperative alterations of the deltoid muscle and the clinical outcomes between the two groups. Statistical significance was set at $p<0.05$ with $95 \%$ confidence intervals. All statistical tests were performed using IBM SPSS for Windows ver. 25.0 (IBM Corp., Armonk, NY, USA).

\section{Results}

No statistically significant differences were observed between the NMES and TENS group when comparing pain VAS, DASH score, ROM, and abduction strength (peak torque) at 12 weeks after surgery. MRI revealed three retears in the repaired rotator cuff at 12 weeks after surgery (Sugaya type ${ }^{19)}$ IV \& V; one retear in NMES group, two retears in TENS group) (Table 2).

Good interobserver reliability was obtained for measurement of thickness and area of the deltoid muscle on MRI (correlation coefficients 0.84 and 0.75 , respectively).

The mean postoperative thickness of the anterior and posterior deltoid was significantly thinner than the mean preoperative thickness ( $p=0.005$ and $p=0.018$, respectively). The mean postoperative thickness of the lateral deltoid was not significantly different from the preoperative thickness $(p=0.324)$. The mean postoperative area of the deltoid was significantly smaller than the mean preoperative area at the level just below the coracoid process $(p=0.001)$ and at the level of the inferior glenoid tubercle $(p=0.008$; Table 3$)$.

Postoperative decrease in the thickness of the anterior, middle, and posterior deltoid at the level just below the cora-

Table 2. Comparison of Postoperative Results between NMES and TENS Groups

\begin{tabular}{lrcc}
\hline \multicolumn{1}{c}{ Variable } & NMES group & TENS group & $p$-value \\
\hline At 6 weeks after surgery & & & \\
\hline Pain VAS & $3.5 \pm 2.7$ & $4.1 \pm 1.5$ & 0.523 \\
\hline DASH score & $45.6 \pm 22.0$ & $46.7 \pm 22.0$ & 0.922 \\
\hline At 12 weeks after surgery & & & \\
\hline Pain VAS & $2.6 \pm 2.5$ & $3.7 \pm 2.1$ & 0.379 \\
\hline DASH score & $29.3 \pm 18.7$ & $30.3 \pm 9.2$ & 0.897 \\
\hline ROM (degrees) & & & \\
\hline Forward Flexion & $149.4 \pm 25.1$ & $156.4 \pm 8.5$ & 0.493 \\
\hline External Rotation & $57.5 \pm 18.3$ & $58.6 \pm 10.3$ & 0.482 \\
\hline Abduction & $150.0 \pm 32.2$ & $159.3 \pm 11.0$ & 0.890 \\
\hline Peak torque, abduction $(\mathrm{Nm})$ & $13.3 \pm 16.4$ & $9.9 \pm 11.4$ & 0.671 \\
\hline Retear & 1 & 2 & 0.522 \\
\hline
\end{tabular}

Values are presented as mean \pm standard deviation or number only.

NMES: neuromuscular electrical stimulation, TENS: transcutaneous electrical nerve stimulation, VAS: visual analogue scale, DASH: Disabilities of the Arm, Shoulder and Hand, ROM: range of motion.

${ }^{\star}$ Sugaya type IV \& $V^{19)}$ coid was $-2.5 \%,-0.7 \%$, and $-6.8 \%$, respectively, in the NMES group, and $-14.0 \%,-2.6 \%$, and $-8.2 \%$, respectively, in the TENS group. Significant difference was obtained in the thickness of the anterior deltoid between the two groups ( $p=0.016$; Fig. 5). However, there was no significant difference in the thickness of the lateral and posterior deltoids between the groups $(p=0.677$ and $p=0.791$, respectively). The postoperative decrease of the deltoid area was $-7.2 \%$ in the NMES group and $-10.1 \%$ in the TENS group, which was not significantly different $(p=0.657$; Fig. 6). However, at the level of the inferior glenoid tubercle, the postoperative decrease in area of the deltoid was $-5.4 \%$ in the NMES group and $-14.0 \%$ in the TENS group, which was significantly different $(p=0.045)$.

Table 3. Decrease of Deltoid Volume at 12 Weeks after Surgery in all Patients

\begin{tabular}{|c|c|c|c|}
\hline Variable & Before surgery & 12 weeks after surgery & $p$-value \\
\hline \multicolumn{4}{|c|}{ Deltoid thickness (mm) } \\
\hline Anterior & $16.0 \pm 4.6$ & $14.7 \pm 4.6$ & 0.005 \\
\hline Lateral & $9.6 \pm 2.5$ & $9.3 \pm 2.0$ & 0.324 \\
\hline Posterior & $20.8 \pm 4.0$ & $19.2 \pm 4.3$ & 0.018 \\
\hline Deltoid area* $^{*}\left(\mathrm{~mm}^{2}\right)$ & $2789.9 \pm 655.2$ & $2552.4 \pm 779.2$ & 0.001 \\
\hline Deltoid area $^{\dagger}\left(\mathrm{mm}^{2}\right)$ & $3,152.37 \pm 715.67$ & $2,904.89 \pm 707.82$ & 0.008 \\
\hline
\end{tabular}

Values are presented as mean \pm standard deviation.

${ }^{*}$ At the level below the coracoid. ${ }^{\dagger}$ At the level of inferior glenoid tubercle.

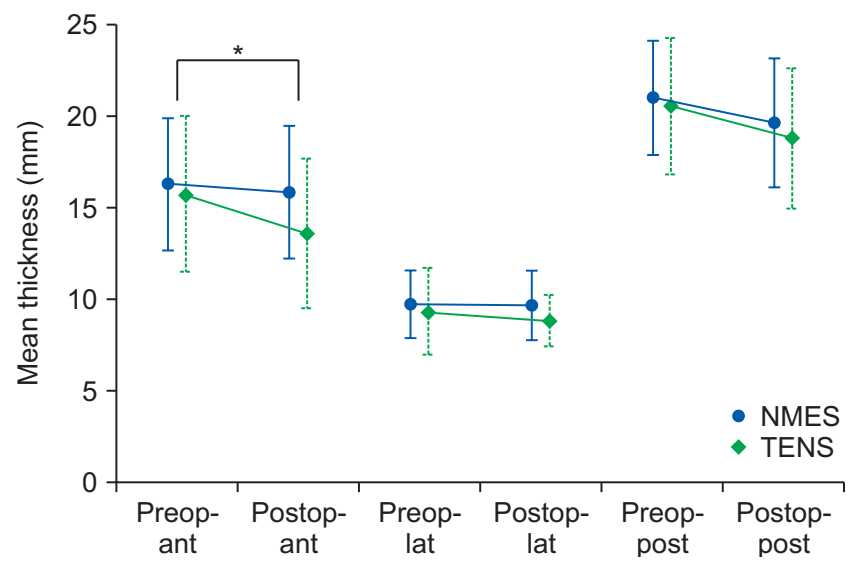

Fig. 5. At the level just below the coracoid, the postoperative decrease in the thickness of the anterior, middle, and posterior deltoid was $-2.5 \%,-0.7 \%$, and $-6.8 \%$, respectively, in the neuromuscular electrical stimulation (NMES) group, and $-14.0 \%,-2.6 \%$, and $-8.2 \%$, respectively, in the transcutaneous electrical nerve stimulation (TENS) group $(p=0.016, p=0.677$, and $p=0.791$, respectively). The difference in the thickness of anterior deltoid between the two groups was statistically significant.

Preop-ant: preoperative anterior thickness of deltoid, Postop-ant: postoperative anterior thickness of deltoid, Preop-lat: preoperative lateral thickness of deltoid, Postop-lat: postoperative lateral thickness of deltoid, Preop-post: preoperative posterior thickness of deltoid, Postop-post: postoperative posterior thickness of deltoid.

*Significant difference between two groups $(p=0.015)$. 
A

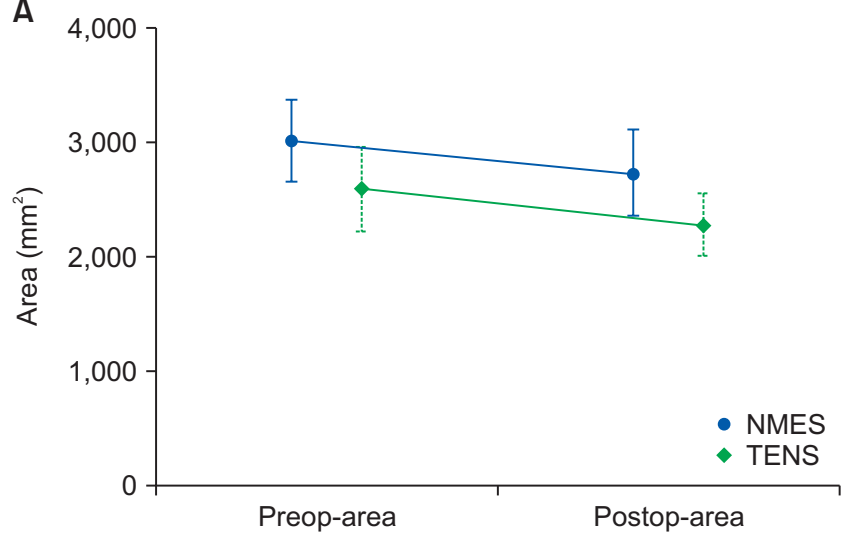

B

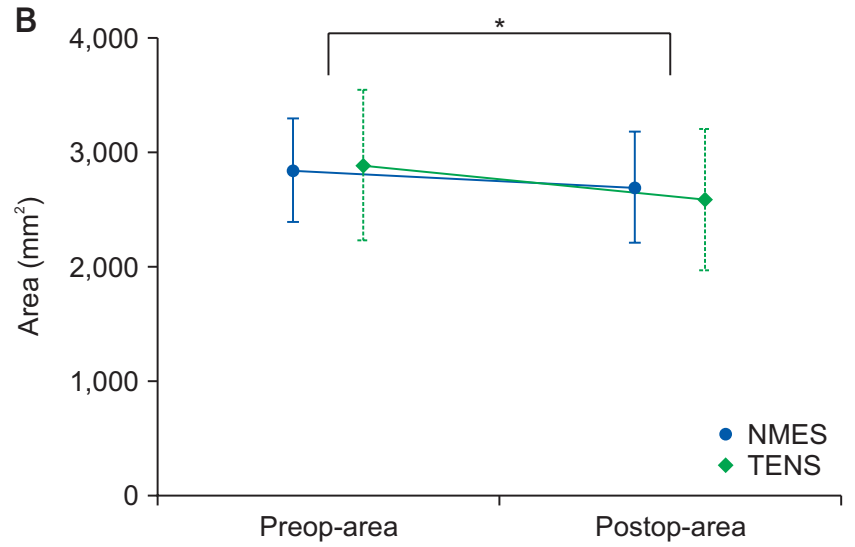

Fig. 6. Postoperative decrease in cross-sectional area of the deltoid muscle in the neuromuscular electrical stimulation (NMES) group and transcutaneous electrical nerve stimulation (TENS) group. (A) At the level just below the coracoid, the postoperative decrease in area of the deltoid was $-7.2 \%$ in the NMES group and $-10.1 \%$ in the TENS group, which was significantly not different $(p=0.657)$. (B) At the level of inferior glenoid tubercle, the postoperative decrease in crosssectional area of the deltoid was $-5.4 \%$ in the NMES group and $-14.0 \%$ in the TENS group, which was significantly different $(p=0.045)$.

Preop-area: preoperative area of deltoid, Postop-area: postoperative area of deltoid.

*Significantly difference between two groups $(p<0.05)$.

\section{Discussion}

Results of this study reveal a significant decrease in anterior deltoid muscle thickness and deltoid area in the NMES group after rotator cuff repair as compared to the TENS group, suggesting that early postoperative application might be useful for reducing atrophy of the deltoid muscle. The TENS group was employed as a control group, intended to allow blinding of patients by providing sham stimulation (placebo stimulation).

Subsequent to rotator cuff repair, it is common to immobilize the shoulder for 6 weeks with an abduction brace, and commence muscle strengthening at 12 weeks. We deliberated that this rehabilitation protocol is likely to cause atrophy of the muscles around the shoulders, as has been indicated in few studies on deltoid atrophy after rotator cuff repair: Cho et al. ${ }^{7}$ reported a $7.3 \%$ decrease in the thickness of the anterior deltoid after open rotator cuff repair; Hata et al. ${ }^{8)}$ reported that mean thickness of the anterior deltoid decreases by $11.4 \%$ (18.4 to $16.3 \mathrm{~mm}$ ) at 6 months and $15.2 \%$ (18.4 to $15.6 \mathrm{~mm}$ ) at 12 months postoperatively, and is significantly smaller than it was prior to surgery $(p<0.05)$. In the present study, the mean thickness of the anterior deltoid decreased by $2.5 \%$ in the NMES group and by $14.0 \%$ in the TENS group at the level just below the coracoid, which was significantly different. At the level of the inferior glenoid tubercle, the mean cross-sectional area of the deltoid decreased by $5.4 \%$ in the NMES group and $14.0 \%$ in the TENS group, which was also significantly different. However, at the level below the coracoid, the mean cross-sectional area of the deltoid decreased by $7.2 \%$ in the NMES group and $10.1 \%$ in the TENS group, which was not significantly different $(p=0.657)$. Taken together, these results indicate that application of the electric stimulation device to the lower part of the deltoid muscle is likely to have caused an effect.

Turkmen and Altun ${ }^{20)}$ reported that increasing the deltoid muscle volume positively affects functional outcomes after arthroscopic rotator cuff repair. Hata et al..$^{8)}$ reported that the reduced deltoid muscle showed no improvement after 1 year, suggesting the importance of preventing atrophy of the deltoid muscle after surgery. Many reports pertaining to the knee have been published, wherein NMES has been reported to be effective in preventing atrophy and weakness of the quadriceps muscle after anterior cruciate ligament surgery. ${ }^{10-15)}$ In the present study, anterior thickness and inferior cross-sectional area of the deltoid muscle were larger than those of the control group; however, unlike previous reports, the clinical outcomes were not significantly different between the groups in our study. This is probably due to the small sample size and short follow-up period.

In the current study, there were two retears in the TENS group and one in the NMES group after rotator cuff repair, all of which occurred early in the study. Isometric evaluation of the deltoid muscle was performed at 6 and 12 weeks after surgery, and we believe that this early muscle strength evaluation had a negative effect. Hence, muscle strength evaluation was subsequently excluded at 6 weeks postoperatively, and there were no further cases of retear. The mean peak torque value of abduction strength was significantly not different between the two groups, but the mean value trended to increase $(9.2 \mathrm{Nm}$ to $13.3 \mathrm{Nm}$ ) in the NMES group, whereas values in the TENS group showed a tendency to decrease $(12.5 \mathrm{Nm}$ to $9.9 \mathrm{Nm}$ ). In a subsequent study, we intend to recruit additional patients and measure the isokinetic strength at 12, 24, 48 weeks after surgery. There was no significant difference in clinical outcomes at 12 weeks after surgery; however, Mahure et al..$^{17)}$ reported that the use of TENS 
significantly reduces opioid use by $>25 \%$ at both 48 hours and 1 week after arthroscopic rotator cuff repair, with statistically significant reductions in the pain scores during the same period.

The results of our study suggest that NMES could be useful in preventing anterior and inferior deltoid atrophy after various shoulder surgeries, such as replacement arthroplasty and fractures, and will have a positive effect on rehabilitation after shoulder surgery.

Our study has several strengths. First, all surgeries were performed by a single surgeon using the suture bridge technique, and all patients were subjected to the same rehabilitation protocol, performed by a single rehabilitation physician. We also performed radiological evaluations using the same MRI scan in the same position. Second, this study was a prospective and randomized controlled trial, and we employed placebo stimulation by TENS for single-blind trial. Third, this study was the first intervention study that prevented muscle atrophy after rotator cuff repair.

The present study also has a few limitations. First, this study had a 3-month follow-up period. We thought that deltoid atrophy was most likely to be prominent at 12 weeks after surgery, before the start of muscle strengthening; hence, postoperative MRI was performed at 12 weeks. However, comparison of clinical outcome seems to be insufficient after 12 weeks. In a followup study, we intend to compare clinical outcomes at 6 and 12 months after surgery, and the final muscle thickness will also be compared at 12 months through MRI or computed tomography. Second, this study had a limited sample size for comparing the deltoid area. The sample size obtained from $\mathrm{G}$ power analysis was 11 per group (alpha error: 0.05, power: 0.80, calculated actual power: 0.82 , total sample size: 22 ). However, only 16 patients were finally included in the analysis, after 4 patients were excluded as per the selection criteria and 2 were lost to followup. Third, the frequency and duration of machine use were evaluated as per the report submitted by patients. Most patients believed that electrical stimulation was helpful for the treatment, and the stimulation devices were portable and easily applicable. Since none of the patients reported that the stimulation device was utilized less than once a day, we, therefore, assessed that stimulation was done properly.

\section{Conclusion}

Results from this prospective single-blind randomized trial demonstrate that compared with TENS, NMES is more efficient in reducing deltoid atrophy after arthroscopic rotator cuff repair, thereby suggesting that NMES has the potential to minimize postoperative atrophy after diverse shoulder surgeries.

\section{References}

1. McElvany MD, McGoldrick E, Gee AO, Neradilek MB, Matsen FA 3rd. Rotator cuff repair: published evidence on factors associated with repair integrity and clinical outcome. Am J Sports Med. 2015;43(2):491-500. doi: 10.1177/0363546514529644.

2. Randelli P, Spennacchio P, Ragone V, Arrigoni P, Casella A, Cabitza P. Complications associated with arthroscopic rotator cuff repair: a literature review. Musculoskelet Surg. 2012;96(1):9-16. doi: 10.1007/s12306-011-0175-y.

3. Galatz LM, Ball CM, Teefey SA, Middleton WD, Yamaguchi K. The outcome and repair integrity of completely arthroscopically repaired large and massive rotator cuff tears. J Bone Joint Surg Am. 2004;86(2):219-24. doi: 10.2106/00004623200402000-00002.

4. Lafosse L, Brozska R, Toussaint B, Gobezie R. The outcome and structural integrity of arthroscopic rotator cuff repair with use of the double-row suture anchor technique. J Bone Joint Surg Am. 2007;89(7):1533-41. doi: 10.2106/JBJS.F.00305.

5. Huberty DP, Schoolfield JD, Brady PC, Vadala AP, Arrigoni P, Burkhart SS. Incidence and treatment of postoperative stiffness following arthroscopic rotator cuff repair. Arthroscopy. 2009;25(8):880-90. doi: 10.1016/j.arthro.2009.01.018.

6. Chung SW, Huong CB, Kim SH, Oh JH. Shoulder stiffness after rotator cuff repair: risk factors and influence on outcome. Arthroscopy. 2013;29(2):290-300. doi: 10.1016/j.arthro. 2012.08.023.

7. Cho NS, Cha SW, Rhee YG. Alterations of the deltoid muscle after open versus arthroscopic rotator cuff repair. Am J Sports Med. 2015;43(12):2927-34. doi: 10.1177/ 0363546515603063.

8. Hata Y, Saitoh S, Murakami N, Kobayashi H, Takaoka K. Atrophy of the deltoid muscle following rotator cuff surgery. J Bone Joint Surg Am. 2004;86(7):1414-9. doi: 10.2106/00004623200407000-00008.

9. Burkhead WZ Jr, Scheinberg RR, Box G. Surgical anatomy of the axillary nerve. J Shoulder Elbow Surg. 1992;1(1):31-6. doi: 10.1016/S1058-2746(09)80014-1.

10. Fitzgerald GK, Piva SR, Irrgang JJ. A modified neuromuscular electrical stimulation protocol for quadriceps strength training following anterior cruciate ligament reconstruction. J Orthop Sports Phys Ther. 2003;33(9):492-501. doi: 10.2519/jospt. 2003.33.9.492.

11. Ediz L, Ceylan MF, Turktas U, Yanmis I, Hiz O. A randomized controlled trial of electrostimulation effects on effussion, swelling and pain recovery after anterior cruciate ligament reconstruction: a pilot study. Clin Rehabil. 2012;26(5):413-22. doi: 10.1177/0269215511421029.

12. Glaviano NR, Saliba S. Can the use of neuromuscular electrical stimulation be improved to optimize quadriceps strengthening? Sports Health. 2016;8(1):79-85. doi: 10.1177/ 


$$
1941738115618174 .
$$

13. Hasegawa S, Kobayashi M, Arai R, Tamaki A, Nakamura T, Moritani T. Effect of early implementation of electrical muscle stimulation to prevent muscle atrophy and weakness in patients after anterior cruciate ligament reconstruction. J Electromyogr Kinesiol. 2011;21(4):622-30. doi: 10.1016/ j.jelekin.2011.01.005.

14. Currier DP, Ray JM, Nyland J, Rooney JG, Noteboom JT, Kellogg R. Effects of electrical and electromagnetic stimulation after anterior cruciate ligament reconstruction. J Orthop Sports Phys Ther. 1993;17(4):177-84. doi: 10.2519/jospt. 1993.17.4.177.

15. Feil S, Newell J, Minogue C, Paessler HH. The effectiveness of supplementing a standard rehabilitation program with superimposed neuromuscular electrical stimulation after anterior cruciate ligament reconstruction: a prospective, randomized, single-blind study. Am J Sports Med. 2011;39(6):1238-47. doi: 10.1177/0363546510396180.

16. Moher D, Hopewell S, Schulz KF, et al. CONSORT 2010 explanation and elaboration: updated guidelines for reporting parallel group randomised trials. BMJ. 2010;340:c869. doi: 10.1136/bmj.c869.

17. Mahure SA, Rokito AS, Kwon YW. Transcutaneous electrical nerve stimulation for postoperative pain relief after arthroscopic rotator cuff repair: a prospective double-blinded randomized trial. J Shoulder Elbow Surg. 2017;26(9):1508-13. doi: 10.1016/j.jse.2017.05.030.

18. Fuchs B, Weishaupt D, Zanetti M, Hodler J, Gerber C. Fatty degeneration of the muscles of the rotator cuff: assessment by computed tomography versus magnetic resonance imaging. J Shoulder Elbow Surg. 1999;8(6):599-605. doi: 10.1016/ s1058-2746(99)90097-6.

19. Sugaya H, Maeda K, Matsuki K, Moriishi J. Repair integrity and functional outcome after arthroscopic double-row rotator cuff repair. A prospective outcome study. J Bone Joint Surg Am. 2007;89(5):953-60. doi: 10.2106/JBJS.F.00512.

20. Turkmen I, Altun G. Increasing the deltoid muscle volume positively affects functional outcomes after arthroscopic rotator cuff repair. Knee Surg Sports Traumatol Arthrosc. 2019;27(1):259-66. doi: 10.1007/s00167-018-5135-8. 